\title{
Five-Year Follow-Up on the Prevalence and Intensity of Infections of Schistosoma mansoni in a Hard-to-Reach District of Madagascar
}

\author{
Stephen A. Spencer, ${ }^{1,2 \star}$ Cortland Linder, ${ }^{2}$ James M. StJ. Penney, ${ }^{2}$ Hannah J. Russell, ${ }^{2}$ Kate Hyde,${ }^{2}$ Caitlin Sheehy, ${ }^{2}$ Alice Reid, ${ }^{3}$ \\ Emmanuel H. Andriamasy, ${ }^{4,5}$ Gina U. Raderalazasoa, ${ }^{5}$ Daniel A. L. Rakotomampianina, ${ }^{5}$ Anjara M. Nandimbiniaina, ${ }^{5}$ \\ Tahiry N. Ranaivoson, ${ }^{5}$ Antsa Andrianiaina, ${ }^{5}$ Sheena M. Cruickshank, ${ }^{5}$ Amaya L. Bustinduy, ${ }^{6}$ J. Russell Stothard, ${ }^{3}$ \\ Glenn T. Edosoa, ${ }^{7,8}$ and Alain M. Rahetilahy ${ }^{8}$ on behalf of MADEX \\ ${ }^{1}$ Royal United Hospitals Bath NHS Foundation Trust, Bath, United Kingdom; ${ }^{2}$ The University of Manchester Faculty of Biology Medicine and \\ Health, Manchester Academic Health Centre, Manchester, United Kingdom; ${ }^{3}$ Unité d'Epidémiologie et de Recherche Clinique, Institut Pasteur de \\ Madagascar, Antananarivo, Madagascar; ${ }^{4}$ Faculté de Médecine, Université d'Antananarivo, Antananarivo, Madagascar; ${ }^{5}$ Department of Clinical \\ Research, London School of Hygiene and Tropical Medicine, London, United Kingdom; ${ }^{6}$ Department of Tropical Disease Biology, Liverpool \\ School of Tropical Medicine, Liverpool, United Kingdom; ${ }^{7}$ World Health Organization, Madagascar Country Office, Antananarivo, Madagascar; \\ ${ }^{8}$ Ministère de la Santé Publique de Madagascar, Antananarivo, Madagascar
}

\begin{abstract}
Schistosomiasis is a major public health problem in Madagascar. The WHO recommends preventive chemotherapy by mass drug administration (MDA) with praziquantel as the primary approach to control Schistosoma mansoni-related morbidity in endemic populations, alongside complementary interventions such as health education. The impact of annual MDA and health education programs was assessed in the hard-to-reach Marolambo district of eastern Madagascar, an area endemic for S. mansoni. Repeated cross-sectional studies undertaken 2015-2019 examined between 300 and 381 school-aged children (aged 5-14 years) annually. The prevalence and infection intensity of S. mansoni were assessed by urine-circulating cathodic antigen (CCA) dipsticks and coproscopy using Kato-Katz (KK) methodologies. After four rounds of annual MDA, a reduction in S. mansoni prevalence was seen in CCA (93.9\% in year $1-87.7 \%$ in year $5 ; P=0.007)$ and $\mathrm{KK}(73.9 \%$ in year $1-59.4 \%$ in year $5 ; P<0.0001)$. The prevalence of heavy-intensity infections roughly halved from $23.7 \%$ to $10.1 \%(P<0.0001)$, and the mean intensity of infection fell by $55.0 \%(480.2-216.3$ eggs per gram of feces). A malacological survey found Biomphalaria pfeifferi snail intermediate hosts in multiple water contact sites including rice paddies, streams, and Nosivolo River. Despite reductions in infection prevalence and intensity, schistosomiasis still poses a significant public health challenge in Marolambo district. Twice yearly MDA cycles and/or community-wide MDA are suggested to better reduce infections. Expanding health education, improving standards of water, sanitation and hygiene, and attention on snail-related control will also be important, especially in rice paddy irrigated areas.
\end{abstract}

\section{INTRODUCTION}

Schistosomiasis is a neglected tropical disease (NTD), which affects 290 million people worldwide across 78 endemic countries. ${ }^{1,2}$ The disease costs more than 200,000 lives and between 1.1 and 3.2 million disability-adjusted life years annually, predominantly in sub-Saharan Africa. ${ }^{3}$ In Madagascar, 107 of 114 districts are endemic with either or both forms of schistosomiasis. ${ }^{4}$ Schistosoma haematobium is the causative species of urogenital schistosomiasis and inflicts disease in northern and western districts of Madagascar, whereas S. mansoni, which causes intestinal schistosomiasis, dominates in southern and eastern districts. ${ }^{5}$ This geographical distribution tracks each trematode's obligatory intermediate snail hosts, Bulinus and Biomphalaria, respectively. ${ }^{6}$ Infection by either $S$. mansoni or $S$. haematobium can lead to significant illness and disability including anemia, undernutrition, reduced exercise tolerance, stunting, wasting, and cognitive impairment and educational loss. ${ }^{2,7}$ Chronic schistosomiasis can result in potentially fatal conditions such as liver fibrosis due to $S$. mansoni in hepatosplenic schistosomiasis, and bladder cancer due to $S$. haematobium with chronic urogenital schistosomiasis. ${ }^{8}$ Over time, the severity of disease correlates with elevated infection intensity, which can be determined by counting and calculating the eggs per gram (EPG) as visualized by coproscopy or uroscopy. ${ }^{9}$ Studies have

*Address correspondence to Stephen A. Spencer, Post-Graduate Medical Centre, Royal United Hospital, Combe Park, Bath BA1 3NG, United Kingdom. E-mail: stephenaspencer@doctors.org.uk demonstrated that through treatment with praziquantel, some downstream conditions of intestinal schistosomiasis, including liver fibrosis, can be reversed, alongside decline in the fecal EPG. ${ }^{10-12}$

In areas with high endemicity, characterized by a > 50\% prevalence of schistosomiasis among school-aged children (SAC), the WHO advises preventive chemotherapy by annual mass drug administration (MDA) with praziquantel to the entire community. ${ }^{13}$ The aim is for more than $75 \%$ of SAC at risk of schistosomiasis to receive regular MDA with Praziquantel (PZQ). ${ }^{14}$ In 2017, global PZQ coverage for SAC in need of regular treatment was $70.8 \%$, whereas coverage of at-risk adults was $16.8 \% .^{15}$ In addition to regular MDA, additional supportive interventions are encouraged to reduce transmission: health education and improving access to safe water and sanitation. ${ }^{16}$ The $\mathrm{WHO}$ schistosomiasis strategy strives first for control of disease morbidity as a public health problem (as defined as a prevalence of heavy infection intensities of < $5 \%$ ) and then for elimination of schistosomiasis as a public health problem (heavy infection prevalence $<1 \%$ in all sentinel sites). ${ }^{16}$ Monitoring response to intervention is therefore vital to determine effectiveness and to intensify MDA schedules if required. ${ }^{17}$

Marolambo is a hard-to-reach district in the east of Madagascar, and our first investigations identified $94 \%$ prevalence of $S$. mansoni among SAC and high burden of disease, where $11 \%$ of children have ultrasonographic evidence of liver fibrosis. ${ }^{18,19}$ To the best of our knowledge, regular monitoring of S. mansoni infection among Malagasy communities has not been attempted. On identification of the considerable burden 
of infection in this region, the National Control Program delivered four rounds of annual MDA between 2015 and 2019. Since 2017, an adjunct schistosomiasis education program (SEP) was codeveloped with the community and introduced in the local schools by the MADagascar mEdical eXpeditions' (MADEX) (S. A. Spencer, et al., unpublished data). The study we report here compares $S$. mansoni infection prevalence and intensity of infection over a five-year period (2015-2019) with observations on Biomphalaria, in the Marolambo district, Madagascar, through four repeated cross-sectional studies.

\section{MATERIALS AND METHODS}

Study area and population. Marolambo is a district in the east of Madagascar, with an estimated population of 215,000. The district is remote with a single access road in seasonal disrepair and no roads between villages. Six villages (Marolambo, Ampasimbola, Ambohitelo, Marofatsy, Vohidamba, and Betampona) were purposively selected for their accessibility by foot and links with community collaborators.

Communities in the Marolambo district rely on environmental water from the Nosivolo River for drinking, cooking, washing, and transport. Historically, there have been additional gravitational water supply systems providing water through a series of pipes from streams to the villages. However, these are prone to damage, leaving very few (if any) in the villages today. Between these six villages, there were a total of 27 gravitational water supplies in 2015, and nine by 2018 . It is common practice for children to assist their families in fetching domestic water from the Nosivolo River or surrounding streams.

Before 2015, there had not been an MDA in the Marolambo district since 2008. Since our identification of endemic infection in the region, ${ }^{19}$ yearly MDA with praziquantel has been delivered to all SAC in the six villages since 2015, and attempted across the entire Marolambo district since 2018 by the Madagascar Ministry of Health. Formal SEPs have been implemented annually since 2017 , which help to rectify community knowledge deficits in understanding of intestinal schistosomiasis.

Study design. Repeated cross-sectional studies were carried out in 2015 (baseline/year 1), 2016 (year 2), 2017 (year 3), and 2019 (year 5). Each study was carried out between June and July in six villages in the Marolambo district: Marolambo, Ampasimbola, Ambohitelo, Marofatsy, Vohidamba, and Betampona (Figure 1).

Each year, schistosomiasis prevalence and infection intensity were determined during a four- to six-week MADEX. All school pupils in each village school (one school per village) were assigned a unique identification number and then stratified by age, gender, and location. Following WHO guidelines, a minimum of 50 children were then randomly selected from each school, with approximately equal numbers by age, gender, and school. ${ }^{13}$ The same process was repeated each year of the study.

Three days were spent within each village, each year of the study, fostering sufficient community engagement. The first two days each year were spent on research (parasitological assessment) and education methodology; MDA was delivered after completion of all research methods, on day 3 .

Parasitology. Children were provided with a labeled stool and urine sample container and instructed how to collect stool and urine. Samples were collected the following day and analyzed by the researchers the same day in the field. Urine samples were tested for circulating cathodic antigen (CCA) using a urine-cassette assay (Rapid Medical Diagnostic Tests, Pretoria, South Africa) by trained and experienced technicians. Assays were conducted following the manufacturer's instructions and independently analyzed by two technicians. Circulating cathodic antigen (CCA) results were recorded in two ways: as a binary outcome of negative or positive, and as categories (negative [-], trace positive [tr], single positive [+], double positive $[++]$, or triple positive $[+++]$ ) as per previous studies. $^{20}$

The Kato-Katz methodology (Vestergaard-Frandsen, Lausanne, Switzerland) was used to identify S. mansoni eggs in the stool samples. ${ }^{21}$ Two thick smears of $41.7 \mathrm{mg}$ of feces were prepared from each sample. The total EPG were calculated from each slide. Results were recorded as follows: 1) positive or negative, 2) the mean EPG between the two slides, and 3 ) the WHO classification of infection intensity as light (1-99 EPG), moderate (100-399 EPG), or heavy ( $\geq 400$ EPG). ${ }^{16}$ To ensure quality control, one in every 10 slides were reexamined by experienced scientists at the Institut Pasteur de Madagascar who were blinded to the findings of others. If a difference was identified, the slide was reexamined, and a consensus EPG was reached and infection intensity assigned.

In year 1, because of a local logistic constraint, no KK microscopy was performed in Marolambo. In year 4 , as national control program delivered MDA to this population 1 month before the MADEX surveillance, collected parasitology data were not included in this analysis.

Malacology In year 5, a freshwater malacological survey was carried out where medically important freshwater snails were collected from known water contact sites (river, streams, or rice paddies) in each village (Figure 1). A transect of 10 square meters per water contact site was assessed for the presence of aquatic snails between the hours of 9:00 and 17: 00 . Snails were collected using a handheld sieve and identified by shell morphology at location. All Biomphalaria snails were then placed in transparent trays and kept in direct sunlight to encourage shedding of $S$. mansoni cercariae. The water from shedding trays was analyzed under light microscopy $(\times 20)$ to identify cercariae, allowing snails to be recorded as infected or uninfected. To determine the species of Biomphalaria, DNA was extracted from a sample of Biomphalaria using DNeasy (Qiagen, Hilden, Germany) at Institut Pasteur de Madagascar. Molecular analysis of these samples was later conducted at Liverpool School of Tropical Medicine, whereby Biomphalaria mitochondrial cytochrome oxidase subunit 1 (CO1) gene fragments were amplified by PCR and later DNA sequencing analysis.

Statistical analysis. All results were recorded in the field with paper and uploaded into Microsoft Excel (Redmond, WA) at a later date. Statistical analyses were performed using StataCorp 2017 (Stata Statistical Software 15, StataCorp LLC, College Station, TX). Chi-squared tests were used to assess the crude relationships between the prevalence by KK and CCA with age, year, gender, and location.

Mixed-effects generalized linear models were used to assess the changes in the prevalence of $S$. mansoni by both $\mathrm{KK}$ and CCA. Tests of departure from linear trend were carried out. Spearman's rank correlation coefficient was used to examine the linear trend between EPG and year. Simple linear 
regression analysis was used to examine the association of mean EPG by year, adjusted by location, age, and gender. Results were considered significant if $P<0.05$.

Retrospective post hoc sample size calculations were carried out to be able to detect differences in prevalence by KK for each village. To detect a difference in the prevalence of $35 \%$ from the baseline prevalence of $74 \%,{ }^{22} 31$ study participants were required for each village. To account for nonresponse and for subgroup analyses, this agreed with our initial target of
50 recruited participants for each village as recommended in WHO guidelines. ${ }^{13}$

Consent and permissions. The study was designed with input from health leaders at both a national and regional level. Before research, briefings in the Malagasy language (local dialect) were held with the local leaders, children, and parents with opportunities for questions and comments. Participation information sheets and consent forms were translated into Malagasy in the local dialect and read to parents and children

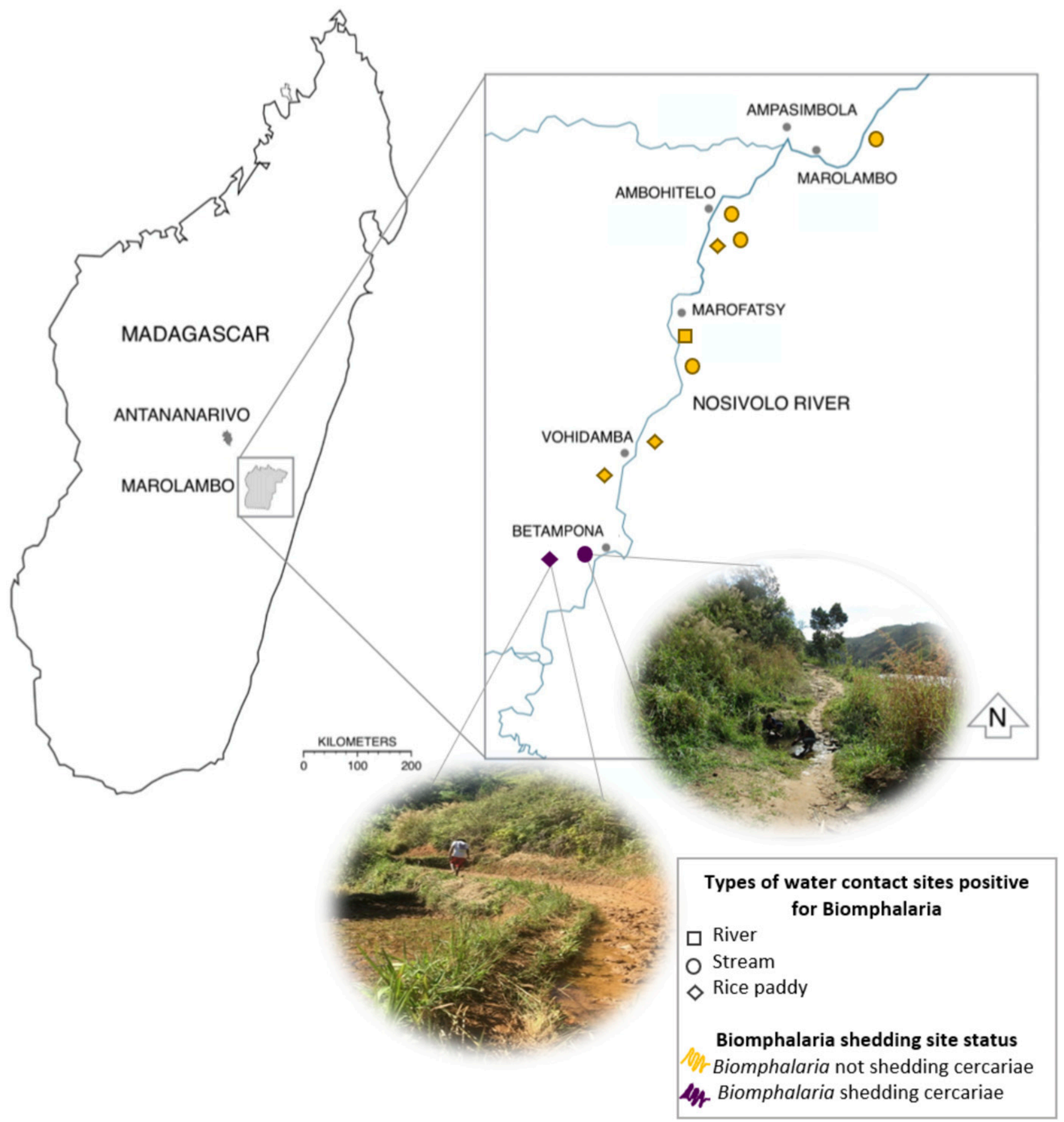

FIGURE 1. Map of Marolambo, Madagascar, with locations of human-water contact sites infested with Biomphalaria snails. This figure appears in color at www.ajtmh.org. 
and the local community. Children could provide assent for the study by stamping their fingerprint in addition to their parents' consent.

Ethical approval. Ethical approval was granted by the University of Manchester Research Ethics Committee and the Ministère de la Santé Publique de Madagascar. Written consent for participation in the study was obtained from the child and their parent/guardian. All human data were anonymized.

\section{RESULTS}

Between 300 and 381 children were recruited each year of the study with $\geq 93.0 \%$ CCA and KK samples to be analyzed from recruited participants each year (Table 1).

Dynamics of S. mansoni prevalence. The baseline prevalence of S. mansoni by CCA was 356/379 (93.9\%) and 215/ 291 (73.9\%) by KK (Figure 2). By CCA, the prevalence reduced in year 3 to $259 / 295\left(87.8 \%\right.$; $P_{\text {adj }} 0.935$ (0.899-0.989), $P=$ 0.007 ; Figure 2). However, there was no further reduction in prevalence by CCA, and in year 5, the prevalence was $321 /$ $366(87.7 \%)$.

By KK, there were no prevalence reductions in years 2 or 3 . However, by year 5 , the prevalence had fallen to $206 / 347$ (59.4\%) ( $\mathrm{PR}_{\mathrm{adj}} 0.809$ [0.719-0.910], $P<0.0001$; Figure 2; see Supplemental Tables S1-S3 for full list of results from the generalized linear mixed models).

There were no differences in prevalence by either CCA or KK between females and males each year of the study (Supplemental Tables S1 and S2).

Dynamics of S. mansoni infection intensity. Comparisons of the changes in prevalence in heavy, moderate, light, and negative infections through $\mathrm{KK}$ analysis demonstrate a district-wide reduction in the prevalence of heavy infections and an increase in the proportion of negative infections, with no significant changes in the prevalence of moderate or light infections (Figure 3). Specifically, the district prevalence of heavy infection intensities fell from 69/291 (23.7\%) to 35/347

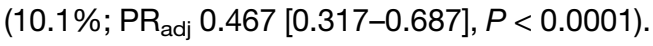

Among infected children, the EPG fell each year (Spearman's rho $=-0.21, P<0.0001)$. The mean EPG fell by $55.0 \%$, from 480.2 at baseline to 216.3 in year 5 (Figure 2). The linear regression analysis estimated a reduction in the district-wide mean EPG of -58.8 (-82.9, -34.6 ; $P<0.0001$; Table 2$)$ following each round of MDA in this study population.

Changes in prevalence and infection intensity stratified by village. When stratified at the level of the village, there were significant reductions in prevalence by KK in Ambohitelo (PR adj 0.665 [0.484-0.912], $P=0.012)$ and Marofatsy $\left(\mathrm{PR}_{\mathrm{adj}} 0.461\right.$ [0.250-0.853], but no other location $(P=$ 0.014 ; Figure 4). When additionally stratified by age, no changes in KK prevalence were observed in any village; however, by CCA, there was a reduction in prevalence among 9- to 12-year-olds in Ambohitelo (Supplemental Figure S1).

Through comparisons of the mean EPG from year 5 to baseline, significant changes were identified in two villages: Ambohitelo (relative reduction of $78.6 \%$ [from 363.0 in year $1-77.8$ in year $5 ; P_{\text {adj }}=0.002$; Table 2]) and Betampona (relative reduction of $43.3 \%$ [from 503.4 in year 1-285.2 in year 5; $\left.P_{\text {adj }}=0.042\right]$ ). Linear regression analyses estimated annual reductions in the mean EPG in children living in only Ambohitelo and Ampasimbola villages (Figure 5).

The scatterplot analysis in Figure 5 demonstrates that there are many outliers with extremely high infection intensities in years 1-3, with a narrowing of EPG data points across the study period, particularly in Ampasimbola, Ambohitelo, Marofatsy, and Vohidamba. However, in year 5 , most of the outliers with extremely high infection intensities originated from either Betampona or Marolambo (Figure 5).

There were no differences in mean EPG between agegroups (Supplemental Figure S2). When additionally stratified by village, the only reduction in mean EPG was observed among 9- to 12-year-olds in Ambohitelo.

Mass drug administration coverage. In year 2, 167/262 (63.7\%) children attended MDA; in year 3, 315/346 (91.0\%) children attended MDA; and in year 4, 222/264 (84.1\%) children attended MDA.

Observations on malacology. Biomphalaria pfeifferi, the intermediate aquatic snail host for $S$. mansoni, was identified in water contact sites in each village, in the Nosivolo River, rice paddies, and streams (Figure 1). Shedding of $S$. mansoni cercariae was seen from $16 / 117(13.7 \%)$ of $B$. pfeifferi

TABLE 1

Study participant characteristics and number recruited each year of the study

\begin{tabular}{|c|c|c|c|c|}
\hline & Baseline/year 1, 2015 & Year 2, 2016 & Year 3, 2017 & Year 5, 2019 \\
\hline & $n(\%)$ & $n(\%)$ & $n(\%)$ & $n(\%)$ \\
\hline \multicolumn{5}{|l|}{ Age-group (years) } \\
\hline $5-8$ & $134(35.2)$ & $108(36.0)$ & $120(40.0)$ & $156(41.8)$ \\
\hline $9-12$ & $161(42.3)$ & $128(42.7)$ & $120(40.0)$ & $150(40.2)$ \\
\hline $13-14$ & $86(22.6)$ & 64 (21.3) & $60(20.0)$ & $67(18.0)$ \\
\hline \multicolumn{5}{|l|}{ Gender } \\
\hline Female & 188 (49.3) & $153(51.0)$ & $149(49.7)$ & $189(50.7)$ \\
\hline Male & $193(50.7)$ & $147(49.0)$ & 151 (50.3) & $184(49.3)$ \\
\hline \multicolumn{5}{|l|}{ Village } \\
\hline Marolambo & $84(22.0)$ & $50(16.7)$ & $50(16.7)$ & 119 (31.9) \\
\hline Ampasimbola & 67 (17.6) & $50(16.7)$ & $50(16.7)$ & $18(4.8)$ \\
\hline Ambohitelo & $69(18.1)$ & $50(16.7)$ & $50(16.7)$ & $59(15.8)$ \\
\hline Marofatsy & $57(15.0)$ & $50(16.7)$ & $50(16.7)$ & $23(6.2)$ \\
\hline Vohidamba & 53 (13.9) & $50(16.7)$ & $50(16.7)$ & $22(5.9)$ \\
\hline Betampona & $51(13.4)$ & $50(16.7)$ & $50(16.7)$ & $132(35.4)$ \\
\hline Total no. of participants & 381 & 300 & 300 & 373 \\
\hline No. eligible CCA samples & $379 / 381$ (99.5) & 298/300 (99.3) & 295/300 (98.3) & $366 / 373$ (98.1) \\
\hline No. eligible KK samples & 291/297 (98.0) & $296 / 300$ (98.7) & $293 / 300$ (97.7) & $347 / 373$ (93.0) \\
\hline
\end{tabular}




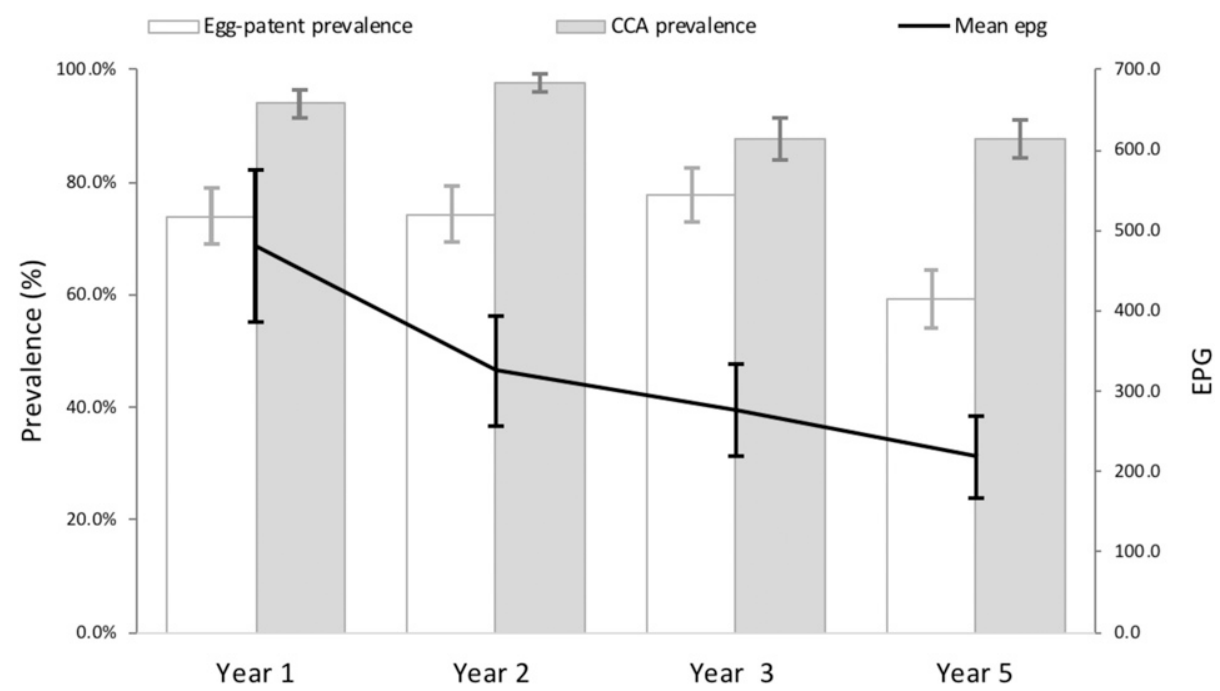

FIGURE 2. Bars representing total prevalence by CCA and Kato-Katz each year of the study, and line graph showing changes in mean EPG of infected individuals. The $95 \% \mathrm{Cl}$ intervals of CCA and Kato-Katz prevalence values and of the mean EPG are shown by vertical error lines.

collected. All B. pfeifferi infected with S. mansoni were found in Betampona village.

On analysis of the mitochondrial CO1, B. pfeifferi was confirmed. All eight Biomphalaria samples that were sequenced were $100 \%$ monomorphic. Inputting this sequence into BLASTN 2.9.0+ showed the sequence to be $100 \%$ identical to B. pfeifferi samples from Zimbabwe (ZWE.236) with $98.1 \%$ sequence identity $B$. pfeifferi from lhosy, Madagascar.
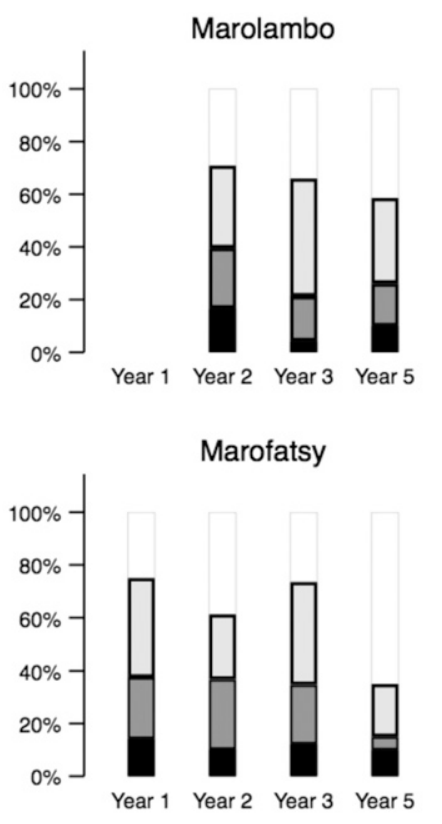

Total

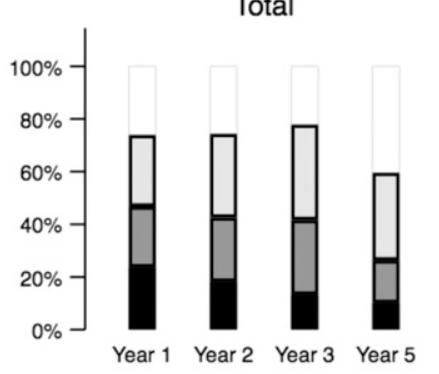

Ampasimbola

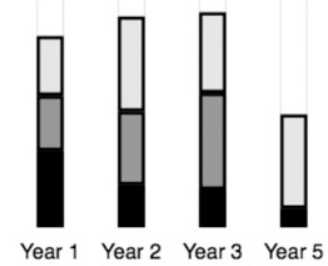

Vohidamba

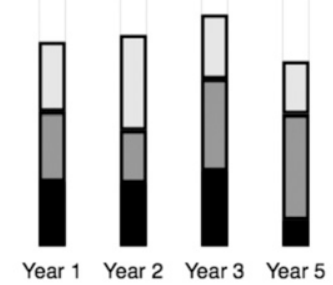

Year 1 Year 2 Year 3 Year 5
Ambohitelo

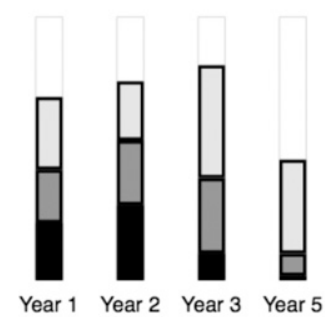

Betampona

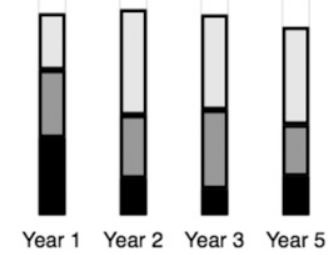

FIGURE 3. Changes in proportion of individuals with each category of infection intensity, ascertained from the Kato-Katz methodology. Black = heavy, dark gray $=$ moderate, light gray $=$ light, and white $=$ negative . 
TABLE 2

Changes in mean EPG across the study period, stratified by location

\begin{tabular}{|c|c|c|c|c|c|c|c|c|c|c|}
\hline \multirow[b]{2}{*}{ Site } & \multicolumn{4}{|c|}{ Mean EPG } & \multicolumn{3}{|c|}{ Year 5 vs. Year 1} & \multicolumn{3}{|c|}{ Linear regression analysis } \\
\hline & Year 1 & Year 2 & Year 3 & Year 5 & Relative change* & $P_{\mathrm{cr}} \dagger$ & $P_{\text {adj }} \dagger$ & Coeff & $95 \% \mathrm{Cl}$ & $P$-value $\neq$ \\
\hline All sites & 480.2 & 325.6 & 276.9 & 216.3 & $-55.0 \%$ & $<0.0001$ & $<0.0001$ & -58.8 & $-82.9,-34.6$ & $<0.0001$ \\
\hline Marolambo & - & 234.4 & 141.3 & 186.8 & $-20.3 \%$ & 0.376 & 0.362 & -8.2 & $-42.0,25.5$ & 0.630 \\
\hline Ampasimbola & 641.1 & 284.4 & 248.4 & 96.0 & $-85.0 \%$ & 0.036 & 0.089 & -146.59 & $-247.5,-45.4$ & 0.005 \\
\hline Ambohitelo & 363.0 & 396.3 & 214.8 & 77.8 & $-78.6 \%$ & 0.001 & 0.002 & -74.1 & $-116.1,-32.3$ & 0.001 \\
\hline Marofatsy & 429.7 & 272.0 & 243.7 & 281.2 & $-34.6 \%$ & 0.287 & 0.277 & -58.3 & $-156.7,40.1$ & 0.124 \\
\hline Vohidamba & 470.6 & 408.0 & 539.4 & 211.7 & $-55.0 \%$ & 0.219 & 0.175 & -47.9 & $-144.8,49.0$ & 0.330 \\
\hline Betampona & 503.4 & 337.2 & 217.3 & 285.2 & $-43.3 \%$ & 0.046 & 0.042 & -41.2 & $-90.2,7.7$ & 0.098 \\
\hline$P($ ANOVA $) \S$ & 0.431 & 0.660 & 0.001 & 0.150 & - & - & - & - & - & - \\
\hline
\end{tabular}

Results from univariable and multivariable linear regression analyses compare the mean EPG from year 5 to year 1 . In addition, this table shows the change in mean EPG for each subsequent year of the study, estimated by the linear regression analysis. Significant $P$-values are shown in bold.

${ }^{*}$ Relative change in mean EPG comparing year 5 with year 1.

† Results from simple univariable (cr) and multivariable linear regression (adj) analyses comparing mean EPG from 2019 to baseline. Multivariable linear regression controlled for the effects of age and gender.

$\ddagger$ Linear regression coefficient and $95 \% \mathrm{Cl}$ of the coefficient: results from the multivariable linear regression model for change in mean EPG for each subsequent year, adjusted by age and gender. $\S$ Results from analysis of variance (ANOVA).

\section{DISCUSSION}

This is, to our knowledge, the first study to describe changes in prevalence and infection intensity of intestinal schistosomiasis in response to ongoing MDA control efforts in Madagascar, conducted with modest resourcing and expedition budgets. Over a five year period (following four rounds of annual school-based MDA with $\mathrm{PZQ}$, and three years of formal schistosomiasis education), the prevalence by CCA assessment demonstrated a small decrease in schistosomiasis prevalence, by $5.7 \%(1.1-10.1 \%)$, whereas there was a reduction in the egg-patent infection prevalence (by KK) of $19.1 \%$ (9.0-28.1\%). Despite these interventions, intestinal schistosomiasis is still a major public health problem, with a final prevalence of $87.7 \%$ by CCA and $59.4 \%$ by KK. Although the decline is important and statistically significant, it will take

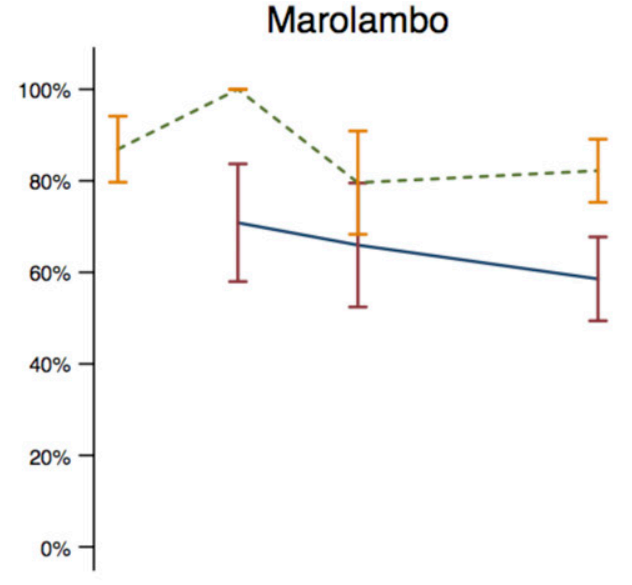

Marofatsy

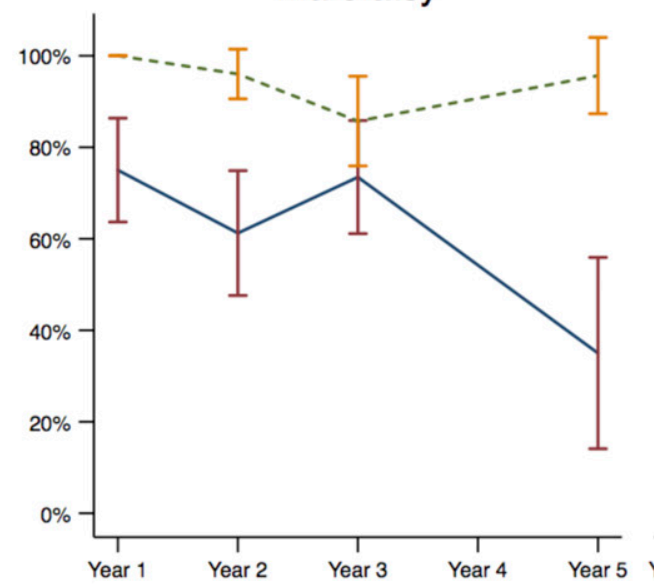

Ampasimbola

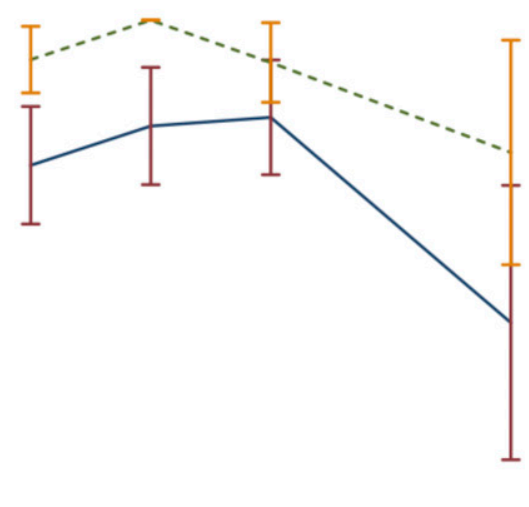

Vohidamba

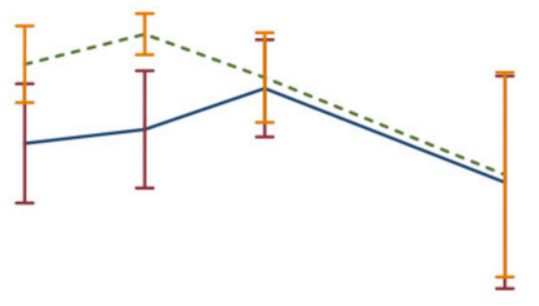

Ambohitelo

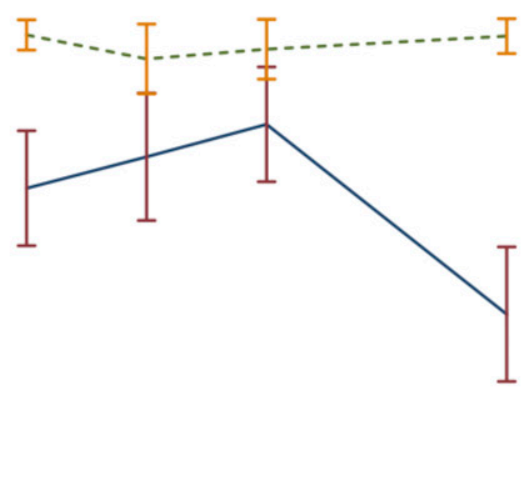

Betampona

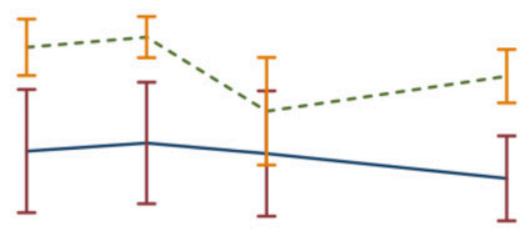

FIGURE 4. Line graphs showing the prevalence of children with S. mansoni by both CCA (dotted lines) and KK (solid lines), for each village. Vertical error lines represent the $95 \% \mathrm{Cl}$ of the prevalence values. This figure appears in color at www.ajtmh.org. 


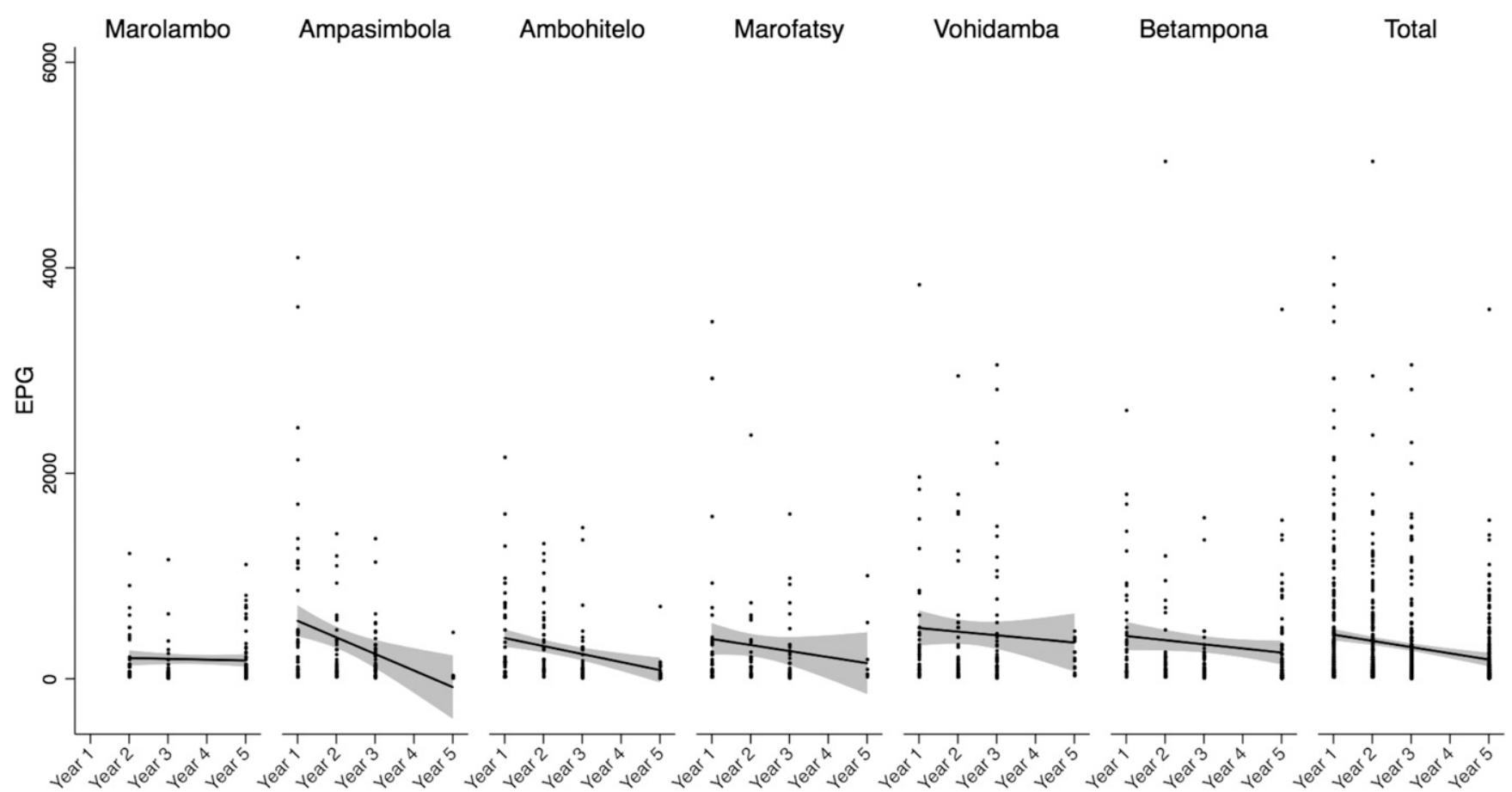

FIGURE 5. Scatterplots of EPG for each location and district total. Lines represent the linear regression plot of mean $\mathrm{EPG}$ with $95 \% \mathrm{Cl}$ (shaded).

much longer than five years of effective annual MDA to gain public health control in this high-endemic and resource-poor setting. Our observations now call for a commensurate future response.

Nonetheless, reductions in the district-level infection intensity observed are more encouraging. The mean EPG fell by $55.0 \%$ (from 480.2 EPG at baseline to 216.2 in year 5), and the prevalence of heavy infection intensities fell by $53.3 \%$ (from $23.7 \%$ to $10.1 \%$ ). Schistosomiasis disease severity is associated with infection intensity, where heavier infections cause more severe disease manifestation. ${ }^{9,23}$ However, light/ moderate intensity infections also cause significant inflammation, leading to pain, diarrhea, undernutrition, cognitive impairment, and fatigue. ${ }^{8,24}$ Although prevalence of moderate and light infections in year 5 was $16.1 \%$ and $33.1 \%$, respectively, these are likely to be underestimates because of the low sensitivity of the KK diagnostic techniques for detecting light intensity infections, ${ }^{25,26}$ and therefore, there could be many more children burdened by schistosomeinduced morbidities which we have seen on ultrasonography. ${ }^{18}$ Studies using refined diagnostic accuracy with novel antigenic measures are therefore needed, with, for example, the promising and highly sensitive, highly specific upconverting particle-lateral flow-circulating anodic antigen assay. 27,28

Our study assessed the impact of MDA and schistosomiasis education over time in Madagascar. Other studies have also examined the response to annual MDA elsewhere with variable and inconsistent findings. Two studies in Kenya and Uganda describe comparable S. mansoni KK baseline prevalence values among selected high-transmission zones (73.5\% and $77.2 \%$, respectively). In Kenya, investigators observed no differences in either prevalence or infection intensity following four rounds of community wide MDA, ${ }^{11}$ whereas in the high prevalence locations of Uganda, investigators reported a reduction in prevalence of $50.1 \%$ after only two rounds of MDA and a reduction of infection intensity by $83.4 \% .{ }^{29}$ On Kome Island, Tanzania, investigators demonstrated a fall in KK prevalence of $S$. mansoni among schoolchildren from $42.2 \%$ to $4.0 \%$ (a $90.5 \%$ reduction) following five years of MDA where they also installed additional safe water supplies. ${ }^{30}$

Persistent hotspots are locations characterized by resilient transmission of schistosomiasis, with persistently high prevalence and/or disease burden despite multiple rounds of effective delivery of MDA, as demonstrated by this study. Whether a community responds to schistosomiasis control efforts is multifactorial; immediate reinfection with schistosomiasis following MDA can be influenced by socioeconomic and water, sanitation, and hygiene (WASH) factors. These include access to latrines, frequency of contact with contaminated waters, and availability of safe drinking water. ${ }^{31}$ For example, in Tanzania, children with no access to drinking water were found to have three-four times the odds of persistent schistosomiasis compared with children with safe access to drinking water, ${ }^{31}$ and a meta-analysis reported that those with access to safe water have $47 \%$ less chance of schistosomiasis than those without access to safe drinking water. $^{32}$ In Marolambo, between 40 and $80 \%$ of schoolchildren are known to have daily contact with the Nosivolo River, and although gravitational water supplies exist, they are prone to damage; very few exist, and the water they carry may not be free from cercariae (S. A. Spencer, et al., unpublished data). Marolambo is a farming district where more than $95 \%$ of the habitants are mixed farmers, many involved in tending rice production. Many children work in the rice paddies, pan for gold in the Nosivolo, and use streams as alternative water sources for washing, drinking, and bathing, noting that children younger than school-age are also infected and at risk (S. A. Spencer, et al., unpublished data); all are areas where 
S. mansoni-infected Biomphalaria were found in this study. The degree of daily exposure to infested waters is therefore likely to be much higher than $40-80 \%$ in this district. Encouraging the use of protective clothing, for example, when farming in rice paddies, would be a good initial measure to prevent long exposure with infected water contact sites. In addition, the low ratio of pit latrines to pupils (two latrines per school) and few household latrines are likely to contribute to the high rates of reinfection, and therefore persistent schistosomiasis. $^{33}$

Biosocial factors are also likely to have an important role in whether a village responds to preventive chemotherapy. ${ }^{34}$ Socioculturally tailored, community participating schistosomiasis education can motivate communities to take responsibility for their own health through behavioral changes and participation in control activities, attendance to MDAs, and promotion of positive transmission and preventionrelated behaviors. In year 3, a SEP was enrolled in the same six schools in Marolambo. This led to increased community rapport with offered preventive chemotherapy witnessed by elevated MDA attendance and latrine use which is likely to have contributed to the reduced prevalence of $S$. mansoni between years 3 and 5 . Our study showed that attendance to MDA increased from $63 \%$ to $91 \%$, reflecting success of the SEP. However, persistent levels of community infection may be sustained by those children missing out on treatment. Population migration from villages outside of the study may have impacted on exposure to MDA, given that total district MDA only started from year 3 .

Schistosomiasis is a focal disease with heterogeneity demonstrated at the community level. ${ }^{35}$ Although our study showed similar levels of schistosomiasis between locations, differences were observed in response to MDA over time. Children in only Ambohitelo village experienced reductions in both the prevalence of infection and infection intensity. Although the largest reduction in egg-patent prevalence was observed in Marofatsy village, there was no significant change in the mean infection intensity. However, the small sample size in Marofatsy in year 5 limits this analysis. Although there were many children with extremely high outlying EPG values in years 1-3 of the study, by year 5 , nearly all outliers came from only two villages: Betampona and Marolambo. These highly infected children are likely to be responsible for major local transmission of $S$. mansoni, which is likely to be one reason that the smallest, albeit nonsignificant, numerical prevalence changes across the study period were observed in these villages. High pretreatment infection intensity is also a recognized risk factor for immediate reinfection following MDA, which may be related to incomplete parasite elimination with praziquantel. ${ }^{36}$ Both the degree of infestation of water contact sites with the intermediate aquatic snail hosts and the prevalence of infected snails are also possible contributing factors as to whether a village responds to MDA. ${ }^{37}$ Although Biomphalaria snails were found in all villages, those with evidence of $S$. mansoni infection were only found in Betampona. This may triangulate evidence on the finding that highly infected individuals were identified in this village and also the largely static high prevalence among children here. The differences in response to MDA between villages may additionally be explained by differences in the number of human-water contact sites between locations and distance of the water from schools. $^{33,34,37}$
The Bill \& Melinda Gates Foundation funded Schistosomiasis Consortium for Operational Research and Evaluation (SCORE) conducted seminal studies where persistent hotspots were defined by village, which over the study period (following four rounds of MDA), the relative prevalence declines by $<35 \%$ and/or the mean infection intensity declines by $<50 \%$. ${ }^{38}$ All six villages surveyed within the Marolambo district represent persistent hotspots by this definition. This issue is not uncommon; in the SCORE studies, more than $30 \%$ of villages were found to be PHSs. ${ }^{33}$

Molecular analysis of $B$. pfeifferi from Marolambo revealed they were $100 \%$ identical to $B$. pfeifferi from Zimbabwe and $98.1 \%$ similar to $B$. pfeifferi from Ihosy, Marolambo. These differences in sequences between $B$. pfeifferi may infer separate invasions of $B$. pfeifferi to Madagascar in recent years. Genetic characterization of $B$. pfeifferi contributes to the understanding of high-risk transmission sites, as $B$. pfeifferi strains vary in their susceptibility to different strains of S. mansoni which have entered into Madagascar from mainland Africa. ${ }^{39}$

Because of early administration of MDA, no reliable S. mansoni parasitological data were collected in year 4 , which is a limitation of this study. In addition, because of the heterogeneity of schistosomiasis infection, findings from this study are not generalizable to other remote districts of Madagascar. However, there is limited access to WASH facilities in many parts of Madagascar. ${ }^{40}$ UNICEF anticipate that across Madagascar, on average, two of five children defecate outdoors, and more than $57 \%$ of people have no access to safe drinking water. ${ }^{41,42}$ This is a particular issue in rural Madagascar, where only $34 \%$ of children have access to safe water. ${ }^{43}$ Children in rural regions who are also endemic with Schistosoma are therefore likely to suffer from high-intensity infections, and therefore, upscaling of national control efforts is required. ${ }^{40}$

\section{CONCLUSION}

Despite four years of preventive chemotherapy in the Marolambo district, Madagascar, intestinal schistosomiasis remains a significant public health challenge, with a prevalence of $86.7 \%$ by CCA and $59.4 \%$ by KK, and $10 \%$ of SAC have heavy infection intensities. This suggests that the burden from schistosomiasis-associated morbidities is still high. Managing high transmission zones where reinfection rates are high can be challenging, as demonstrated in this study, and gaining control with MDA will take much longer than expected. Tackling this issue in hard-to-reach communities living in poverty is necessary to achieve both the WHO road map toward NTD control and elimination and UN agenda for Universal Health Coverage, but this is not possible without sufficient external funding. Vitally, scaling up multi-sectoral efforts is necessary to achieve schistosomiasis control. These efforts should be directed toward improving WASH factors, installing points of access to safe water, expansion of culturally tailored SEPs to motivate communities to take responsibility for their local schistosomiasis control in addition to strategies for snail control. Community-wide, reaching preschool-aged children, and/ or twice yearly MDA is also likely to be beneficial, and its impact should be tested through robust research methodology. 
Received November 6, 2020. Accepted for publication January 10, 2021.

Published online March 8, 2021.

Note: Supplemental tables and figures appear at www.ajtmh.org

Acknowledgments: We first and foremost thank the children and families who participated in this study, and the communities in the Marolambo district for their support and hospitality. We thank Arminder Deol for her advice with statistical analyses. We also thank Ed Wilkins, Bertie Squire, Tony Freemont, Manchester Medical School, Durrell Wildlife Conservation Trust, Scientific Exploration Society, and Rivers Foundation. Study collaborators who deserve sincere thanks and acknowledgment include Isla L. Young, Jemima Henstridge-Blows, Stephanie Jokhan, Heather Lawson, Graham Dodge, Robbie Kornitschky, Benedicte Sjoflot, Daniel Ripley, Lalarizo R. Mahary, Elodie P. Ranjanoro, Rasolofomanana S. M. Michèle, Zafera A. Rohe, Jenny S. Emmanoela, Rasoamanamihaja Clara Fabienne, and Andrianjaka Louis de Gonzague.

Financial support: Biotechnology and Biological Sciences Research Council (BBSRC) school regional champion funds and DTP funds awarded to S. M. C. American Association for the Advancement of Science (AAAS) Leshner fellowship funds awarded to S. M. C. British Society of Immunology Communicating Immunology Grants awarded to S. A. S., S. M. C., K. H., and J. M. St. J. P. Scientific Exploration Society Rivers Award for Health and Humanities awarded to S. A. S. Royal Geographical Society Geographical Fieldwork Grant awarded to S. A.S.

Authors' addresses: Stephen A. Spencer, Cortland Linder, James M. StJ. Penney, Hannah J. Russell, Kate Hyde, Caitlin Sheehy, and Sheena M. Cruickshank, University of Manchester Medical School, Manchester, United Kingdom, E-mails: stephenaspencer@doctors. org.uk, cortlinder@gmail.com, jamespenney111@gmail.com, hannahjrussell@gmail.com, k_hyde@hotmail.co.uk, caitlin.sheehy@ gmail.com, and sheena.cruickshank@manchester.ac.uk. Alice Reid, and J. Russell Stothard, Liverpool School of Tropical Medicine, Liverpool, United Kingdom, E-mails: russell.stothard@Istmed.ac.uk, and alice_reid123@hotmail.com. Emmanuel H. Andriamasy, Gina U. Raderalazasoa, Daniel A. L. Rakotomampianina, Anjara M. Nandimbiniaina, Tahiry N. Ranaivoson, and Antsa Andrianiaina, Faculté de Médecine, Université d'Antananarivo, Antananarivo, Madagascar, E-mails: andriamasyharizaka@gmail.com, gina.raderalazasoa@gmail.com, livadaniel1@gmail.com, njaramihaja@yahoo.fr, andrianiainaantsame@ gmail.com, and ranaivoson.t@yahoo.com. Amaya L. Bustinduy, London School of Hygiene and Tropical Medicine, London, United Kingdom, E-mail: amaya.Bustinduy@Ishtm.ac.uk. Glenn T. Edosoa, and Alain M. Rahetilahy, Ministère de la Santé Publique de Madagascar, Antananarivo, Madagascar, E-mails: edosoag@who.int, and rahetilahyalainmarcel@ gmail.com.

This is an open-access article distributed under the terms of the Creative Commons Attribution (CC-BY) License, which permits unrestricted use, distribution, and reproduction in any medium, provided the original author and source are credited.

\section{REFERENCES}

1. Malecela MN, 2019. Reflections on the decade of the neglected tropical diseases. Int Health 11: 338-340.

2. Ezeamama AE, Bustinduy AL, Nkwata AK, Martinez L, Pabalan N, Boivin MJ, King $\mathrm{CH}, 2018$. Cognitive deficits and educational loss in children with schistosome infection-a systematic review and meta-analysis. PLoS Negl Trop Dis 12: e0005524.

3. DALYs GBD, Collaborators H, 2017. Global, regional, and national disability-adjusted life-years (DALYs) for 333 diseases and injuries and healthy life expectancy (HALE) for 195 countries and territories, 1990-2016: a systematic analysis for the Global Burden of Disease Study 2016. Lancet 390: 1260-1344.

4. Madagascar MdISPd, 2016. Cartographie des Maladies Tropicales Negligees a Chimiotherapie Preventive SchistosomiasisGeo Helminthiases-Filariose Lymphatique. Antananarivo, Madagascar: Ministère de la Santé Publique de Madagascar.

5. Madagascar MdISPd, 2016. Plan Directeur de Lutte Contre les Maladies Tropicales Negligées-(MTN) 2016-2020.
Antananarivo, Madagascar: Ministère de la Santé Publique de Madagascar.

6. Stothard JR, Bremond P, Andriamaro L, Sellin B, Sellin E, Rollinson D, 2001. Bulinus species on Madagascar: molecular evolution, genetic markers and compatibility with Schistosoma haematobium. Parasitology 123 (Suppl): S261-S275.

7. Andrade G, Bertsch DJ, Gazzinelli A, King CH, 2017. Decline in infection-related morbidities following drug-mediated reductions in the intensity of Schistosoma infection: a systematic review and meta-analysis. PLoS Negl Trop Dis 11: e0005372.

8. King $\mathrm{CH}$, Dangerfield-Cha M, 2008. The unacknowledged impact of chronic schistosomiasis. Chronic Illn 4: 65-79.

9. Colley DG, Bustinduy AL, Secor WE, King CH, 2014. Human schistosomiasis. Lancet 383: 2253-2264.

10. Shen $Y$ et al., 2019. Five-year impact of different multi-year mass drug administration strategies on childhood Schistosoma mansoni-associated morbidity: a combined analysis from the schistosomiasis consortium for operational research and evaluation cohort studies in the Lake Victoria regions of Kenya and Tanzania. Am J Trop Med Hyg 101: 1336-1344.

11. Sircar AD, Mwinzi PNM, Onkanga IO, Wiegand RE, Montgomery SP, Secor WE, 2018. Schistosoma mansoni mass drug administration regimens and their effect on morbidity among schoolchildren over a 5-year period-Kenya, 2010-2015. Am J Trop Med Hyg 99: 362-369.

12. Gunda DW, Kilonzo SB, Manyiri PM, Peck RN, Mazigo HD, 2020. Morbidity and mortality due to Schistosoma mansoni related periportal fibrosis: could early diagnosis of varices improve the outcome following available treatment modalities in sub saharan Africa? A scoping review. Trop Med Infect Dis 5: 20.

13. WHO, 2006. Preventive Chemotherapy in Human Helminthiasis: Coordinated Use of Anthelminthic Drugs in Control Interventions: A Manual for Health Professionals and Programme Managers. Geneva, Switzerland: World Health Organization.

14. WHO, 2012. Accelerating Work to Overcome the Global Impact of Neglected Tropical Diseases - a Roadmap for Implementation. Geneva, Switzerland: World Health Organization.

15. WHO, 2020. Update on the Global Status of Implementation of Preventive Chemotherapy (PC). Geneva, Switzerland: World Health Organization.

16. WHO, 2013. Schistosomiasis Progress Report 2001-2011 and Strategic Plan 2012-2020. Geneva, Switzerland: World Health Organization.

17. Deol AK et al., 2019. Schistosomiasis - assessing progress toward the 2020 and 2025 global goals. N Engl J Med 381: 2519-2528.

18. Russell HJ et al., 2020. A cross-sectional study of periportal fibrosis and Schistosoma mansoni infection among school-aged children in a hard-to-reach area of Madagascar. Trans $R$ Soc Trop Med Hyg 114: 315-322.

19. Spencer SA et al., 2017. High burden of Schistosoma mansoni infection in school-aged children in Marolambo district, Madagascar. Parasit Vectors 10: 307.

20. Stothard JR, Kabatereine NB, Tukahebwa EM, Kazibwe F, Rollinson D, Mathieson W, Webster JP, FenwickA, 2006. Use of circulating cathodic antigen (CCA) dipsticks for detection of intestinal and urinary schistosomiasis. Acta Trop 97: 219-228.

21. Katz N, Chaves A, Pellegrino J, 1972. A simple device for quantitative stool thick-smear technique in Schistosomiasis mansoni. Rev Inst Med Trop Sao Paulo 14: 397-400.

22. Kittur N, Binder S, Campbell CH, King CH, Kinung'hi S, Olsen A, Magnussen P, Colley DG, 2017. Defining persistent hotspots: areas that fail to decrease meaningfully in prevalence after multiple years of mass drug administration with praziquantel for control of schistosomiasis. Am J Trop Med Hyg 97: 1810-1817.

23. Bustinduy AL, Thomas CL, Fiutem JJ, Parraga IM, Mungai PL, Muchiri EM, Mutuku F, Kitron U, King CH, 2011. Measuring fitness of Kenyan children with polyparasitic infections using the 20-meter shuttle run test as a morbidity metric. PLoS Negl Trop Dis 5: e1213.

24. King CH, Dickman K, Tisch DJ, 2005. Reassessment of the cost of chronic helmintic infection: a meta-analysis of disabilityrelated outcomes in endemic schistosomiasis. Lancet 365 : 1561-1569. 
25. Barenbold O, Raso G, Coulibaly JT, N'Goran EK, Utzinger J, Vounatsou P, 2017. Estimating sensitivity of the Kato-Katz technique for the diagnosis of Schistosoma mansoni and hookworm in relation to infection intensity. PLoS Negl Trop Dis 11: e0005953.

26. Sousa-Figueiredo JC, Betson M, Kabatereine NB, Stothard JR, 2013. The urine circulating cathodic antigen (CCA) dipstick: a valid substitute for microscopy for mapping and point-of-care diagnosis of intestinal schistosomiasis. PLoS Negl Trop Dis 7: e2008.

27. Corstjens $P$ et al., 2020 Circulating anodic antigen (CAA): a highly sensitive diagnostic biomarker to detect active Schistosoma infections-improvement and use during SCORE. Am J Trop Med Hyg 103 (Suppl 1): 50-57.

28. Sousa MS, van Dam GJ, Pinheiro MCC, de Dood CJ, Peralta JM, Peralta RHS, Daher EF, Corstjens PLAM, Bezerra FSM, 2019. Performance of an ultra-sensitive assay targeting the circulating anodic antigen (CAA) for detection of Schistosoma mansoni infection in a low endemic area in Brazil. Front Immunol 10:682.

29. Zhang Y, Koukounari A, Kabatereine N, Fleming F, Kazibwe F, Tukahebwa E, Stothard JR, Webster JP, Fenwick A, 2007. Parasitological impact of 2-year preventive chemotherapy on schistosomiasis and soil-transmitted helminthiasis in Uganda. BMC Med 5: 27.

30. Kaatano GM et al., 2015. Integrated schistosomiasis and soiltransmitted helminthiasis control over five years on Kome Island, Tanzania. Korean J Parasitol 53: 535-543.

31. Donohue RE, Mashoto KO, Mubyazi GM, Madon S, Malecela MN, Michael E, 2017. Biosocial determinants of persistent schistosomiasis among schoolchildren in Tanzania despite repeated treatment. Trop Med Infect Dis 2: 61.

32. Grimes JE, Croll D, Harrison WE, Utzinger J, Freeman MC, Templeton MR, 2014. The relationship between water, sanitation and schistosomiasis: a systematic review and meta-analysis. PLoS Negl Trop Dis 8: e3296.

33. Kittur $\mathrm{N}$ et al., 2020. Discovering, defining, and summarizing persistent hotspots in SCORE studies. Am J Trop Med Hyg 103: 24-29.

34. Mwanga JR, Kinung'hi SM, Mosha J, Angelo T, Maganga J, Campbell C, 2020. Village response to mass drug administration for schistosomiasis in Mwanza region, northwestern
Tanzania: are we missing socioeconomic, cultural, and political dimensions? Am J Trop Med Hyg 103: 1969-1977.

35. Meurs L, Mbow M, Boon N, van den Broeck F, Vereecken K, Dièye TN, Abatih E, Huyse T, Mboup S, Polman K, 2013. Microgeographical heterogeneity in Schistosoma mansoni and $S$. haematobium infection and morbidity in a co-endemic community in northern Senegal. PLoS Negl Trop Dis 7: e2608.

36. Mbanefo EC, Huy NT, Wadagni AA, Eneanya Cl, Nwaorgu O, Hirayama K, 2014. Host determinants of reinfection with schistosomes in humans: a systematic review and meta-analysis. PLoS Negl Trop Dis 8: e3164.

37. Pennance T, Person B, Muhsin MA, Khamis AN, Muhsin J, Khamis IS, Mohammed KA, Kabole F, Rollinson D, Knopp S, 2016. Urogenital schistosomiasis transmission on Unguja Island, Zanzibar: characterisation of persistent hot-spots. Parasit Vectors 9: 646.

38. Kittur $\mathrm{N}$ et al., 2019. Persistent hotspots in schistosomiasis consortium for operational research and evaluation studies for gaining and sustaining control of schistosomiasis after four years of mass drug administration of praziquantel. Am J Trop Med Hyg 101: 617-627.

39. Campbell G, Jones CS, Lockyer AE, Hughes S, Brown D, Noble LR, Rollinson D, 2000. Molecular evidence supports an African affinity of the neotropical freshwater gastropod, Biomphalaria glabrata, say 1818, an intermediate host for Schistosoma mansoni. Proc Biol Sci 267: 2351-2358.

40. Rasoamanamihaja CF, Rahetilahy AM, Ranjatoarivony B, Dhanani N, Andriamaro L, Andrianarisoa SH, Jourdan PM, 2016. Baseline prevalence and intensity of schistosomiasis at sentinel sites in Madagascar: informing a national control strategy. Parasit Vectors 9: 50

41. Makoni M, 2019. Madagascar's battle for health. Lancet 393: 1189-1190.

42. Institut National de la Statistique (INSTAT), 2019. Madagascar Multiple Indicator Cluster Survey, 2018. Antananarivo, Madagascar: INSTAT, UNICEF, World Bank, SUN, USAID.

43. Ram PK, Kelsey E, Rasoatiana, Miarintsoa RR, Rakotomalala O, Dunston C, Quick RE, 2007. Bringing safe water to remote populations: an evaluation of a portable point-of-use intervention in rural Madagascar. Am J Public Health 97: 398-400. 